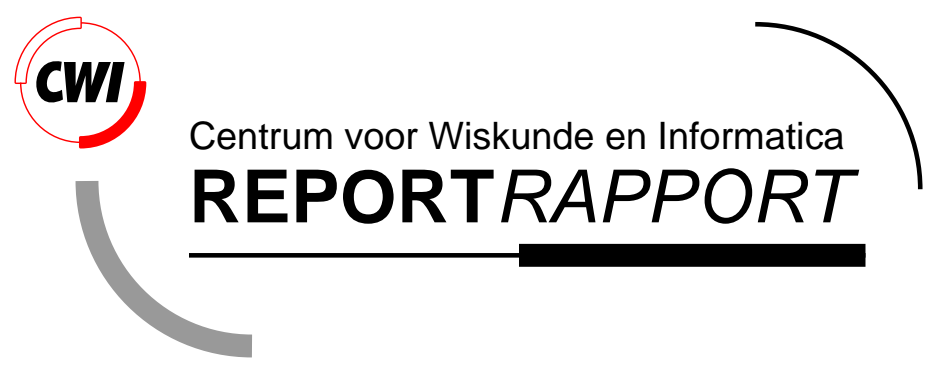

Annular filters for binary images

H.J.A.M. Heijmans and C.Ronse

Department of Operations Reasearch, Statistics, and System Theory

BS-R9604 1996 
Report BS-R9604

ISSN 0924-0659

CWI

P.O. Box 94079

1090 GB Amsterdam

The Netherlands

$\mathrm{CWI}$ is the National Research Institute for Mathematics and Computer Science. CWI is part of the Stichting Mathematisch Centrum (SMC), the Dutch foundation for promotion of mathematics and computer science and their applications.

SMC is sponsored by the Netherlands Organization for Scientific Research (NWO). CWI is a member of ERCIM, the European Research Consortium for Informatics and Mathematics.

Copyright @ Stichting Mathematisch Centrum P.O. Box 94079, 1090 GB Amsterdam (NL) Kruislaan 413, 1098 SJ Amsterdam (NL) Telephone +3120 5929333 Telefax +3120 5924199 


\title{
Annular Filters for Binary Images
}

\author{
Henk J.A.M. Heijmans \\ CWI \\ P.O. Box 94079, 1090 GB Amsterdam, The Netherlands \\ Christian Ronse \\ Université Louis Pasteur \\ LSIIT - URA 1871 \\ Département d'Informatique \\ 7, rue René Descartes, 67084 Strasbourg Cedex, France
}

\begin{abstract}
A binary annular filter removes isolated points in the foreground and the background of an image. Here, the adjective 'isolated' refers to an underlying adjacency relation between pixels, which may be different for foreground and background pixels. In this paper, annular filters are represented in terms of switch pairs. A switch pair consists of two operators which govern the removal of points from foreground and background, respectively. In the case of annular filters, switch pairs are completely determined by foreground and background adjacency. It is shown that a specific triangular condition in terms of both adjacencies is required to establish idempotence of the resulting annular filter.
\end{abstract}

AMS Subject Classification (1991): 68U10

Keywords \& Phrases: image processing, mathematical morphology, annular filter, self-dual (morphological) operator, switch operator, switch pair, adjacency relation, isolated point.

\section{Introduction}

Let $E$ be a Euclidean or discrete space, and take a symmetric structuring element $B$; then the translation invariant set operator on $\mathcal{P}(E)$ given by

$$
X \mapsto X \cap(X \oplus B)
$$

is an algebraic opening in the sense that it is increasing, anti-extensive, and idempotent. This opening is called annular opening. We normally assume that $B$ does not contain the origin 0 of $E$, otherwise this opening reduces to the identity. Annular openings were introduced by Serra $[9$, pp. 107,108], where he illustrated the behaviour of this new type of operator by taking $E=\mathbb{Z}^{2}$ and for $B$ a ring $B=\{b \in E \mid r<d(0, b)<R\}$, where $0<r<R$ and where $d$ is a distance function approximating the Euclidean distance. This opening removes from a set $X$ every point $p$ such that there is no point $q \in X$ verifying $r<d(p, q)<R$. As shown in Figure 5.2 of [9], the 
effect of such an operator is to remove isolated grains in a set. One can for instance choose for $d$ the checkerboard distance, and $0<r<1<R<2$, so that $B$ will be the 8-neighbourhood of the origin; here the annular opening will remove from a set $X$ all points having no 8-neighbour in $X$ (in other words, isolated from the point of view of 8-adjacency).

This example explains the term annular used to designate this opening, in contrast with the usual structural opening by an arbitrary structuring element $B: X \mapsto X \circ B=(X \ominus B) \oplus B$; here every portion of $X$ which is too small (or too narrow) to contain a translate of $B$ is removed. The behaviours of the two types of openings are clearly distinct: with the annular opening, parts of an image are removed on the basis of their isolation, while with the usual structural opening, parts of an image are removed on the basis of their size.

One can consider the dual

$$
X \mapsto X \cup(X \ominus B)
$$

of an annular opening, called the annular closing, whose effect is to add to a set isolated points of the background $X^{\mathrm{c}}$, in other words to remove isolated hole points.

Now the basic feature of annular openings and closings, namely the notion of isolated point, can be defined independently of a structuring element, but simply from an adjacency relation on $E$. We note from the symmetry of $B$ that for $x, y \in E$ we have

$$
x \in B_{y} \Longleftrightarrow y \in B_{x}
$$

this gives thus a symmetric adjacency relation $\sim$ defined by $x \sim y$ iff $x \in B_{y}$, and we say then that $x$ is adjacent to $y$. An isolated point of $X$ is a point in $X$ which is adjacent to no point of $X$. Then it is clear that the behaviour of the above-defined annular opening (resp., closing) is to remove from $X$ its isolated points (resp., to add to $X$ the isolated points of its complement $\left.X^{\mathrm{c}}\right)$.

We generalize this idea as follows. Let $\sim$ be an arbitrary symmetric relation on $E$, which we call adjacency; thus we say that $x$ is adjacent to $y$ when $x \sim y$. Write $\operatorname{adj}(x)$ for the set of points which are adjacent to $x$ :

$$
\operatorname{adj}(x)=\{y \in E \mid x \sim y\}
$$

We call an isolated point of $X$ a point $x \in X$ such that $\operatorname{adj}(x) \cap X=\varnothing$. The adjacency relation $\sim$ gives rise to the dilation $\delta$ given by

$$
\delta(X)=\{h \in E \mid \operatorname{adj}(h) \cap X \neq \varnothing\},
$$

and the dual erosion $\varepsilon$ given by

$$
\varepsilon(X)=\{h \in E \mid \operatorname{adj}(h) \subseteq X\} .
$$

Then the operator

$$
\text { id } \wedge \delta: X \mapsto X \cap \delta(X)
$$

is an opening which removes from $X$ all isolated points, while the operator

$$
\text { id } \vee \varepsilon: X \mapsto X \cup \varepsilon(X)
$$

is the dual closing which adds to $X$ all isolated points of its complement $X^{\mathrm{c}}$.

When $\sim$ is translation-invariant (for all $x, y, p \in E, x \sim y \Longleftrightarrow x+p \sim y+p$ ), taking $B=\operatorname{adj}(0)$, every $x \in E$ gives $\operatorname{adj}(x)=B_{x}$, and we find again Serra's annular opening and closing based on the structuring element $B$. 
The starting point of this paper is the following question: Is it possible to devise an increasing idempotent operator which would behave as both an annular opening and an annular closing, in other words removing from a set all its isolated points, and at the same time adding to it all isolated points of its complement?

In the case of usual morphological openings and closings, we can see that it is generally impossible to combine their behaviours in an idempotent operator. Consider for example an image forming an alternation of dark and light stripes, and a structuring element $A$ wider than these stripes (see Figure 1):
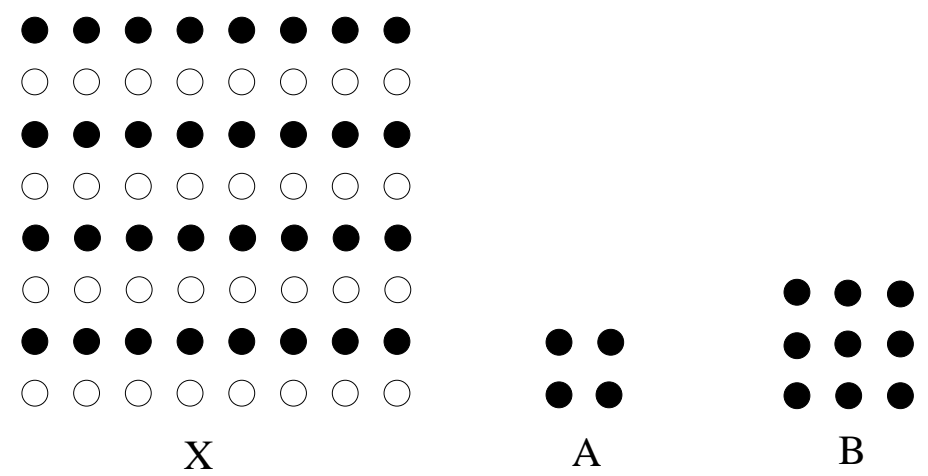

Fig. 1. $X$ is an alternation of black (background) and white (foreground) stripes (infinitely extended). The structuring element $A$ is wider than these stripes and the symmetric square structuring element $B$ has width 3 times that of the stripes.

The structural opening $X \mapsto X \circ A$ removes these white stripes and transforms $X$ into the empty set. Conversely, from the point of view of the structural closing by $A$, image $X$ shows dark stripes on a light background; thus the closing removes these dark stripes and transforms $X$ into the overall set $E$. Therefore the behaviour of the morphological opening and closing are contradictory, and the composition of one by the other gives different results according to which one (opening or closing) is applied first. Note that the median operator using a 3-stripe thick square structuring element $B$ has a paradoxical behaviour combining that of the opening and the closing: it transforms $X$ into its negative, in other words it interchanges foreground and background stripes; this leads to an oscillation of period 2 when we repeat the median operator. We see thus that we cannot build an idempotent operator combining the behaviour of both the structural opening and the structural closing.

However, with annular openings and closings, such a combination is possible: in [3] it was shown that the operator $\psi=(\mathrm{id} \wedge \delta) \vee \varepsilon$, with $\delta, \varepsilon$ given by (1.2)-(1.3) has all the desired properties (removal of isolated points from foreground and background and idempotence) presumed that the following adjacency triple condition is satisfied:

$$
\forall x \in X, \quad \exists y, z \in E, \quad x \sim y \sim z \sim x .
$$

We will show here that this condition is also necessary for idempotence. In the case of translationinvariant operators, the condition (1.6) means that the symmetric structuring element $A$ satisfies

$$
0 \in A \oplus A \oplus A
$$


(or equivalently $A \cap(A \oplus A) \neq \varnothing$ ); it was then shown in [3] that the operator $\psi$ given by

$$
\psi(X)=[X \cap(X \oplus A)] \cup(X \ominus A)=[X \cup(X \ominus A)] \cap(X \oplus A)
$$

is a self-dual morphological filter. This filter is called annular filter. In this paper we will extend the results in [3] by choosing two adjacency relations $\stackrel{1}{\sim}$ and $\stackrel{0}{\sim}$, called foreground adjacency and background adjacency, from which we derive neighbourhoods $\operatorname{adj}_{1}(x)$ and $\operatorname{adj}_{0}(x)$ of a point $x$ as in (1.1). The two adjacency relations need not be the same: this is for example the case in the digital topology of $\mathbb{Z}^{2}$, where we have opposite choices of adjacencies (4- and 8-adjacency) for figure and complement. Now the adjacencies can be used for testing the isolation of points of the figure and the complement respectively: an isolated point of $X$ is a point $x \in X$ such that no $y \in X$ satisfies $x \stackrel{1}{\sim} y$ (in brief, $\operatorname{adj}_{1}(x) \cap X=\varnothing$ ), while an isolated point of $X^{\mathrm{c}}$ is a point $x \in X^{\mathrm{c}}$ for which there is no $y \in X^{\mathrm{c}}$ with $x \stackrel{0}{\sim} y$ (in $\operatorname{brief}_{\operatorname{adj}_{0}}(x) \cap X^{\mathrm{c}}=\varnothing$ ).

The following example shows that the choice of adjacency relations cannot be arbitrary. Let $E=\mathbb{Z}^{2}$, and define $x \stackrel{1}{\sim} y$ if $x$ and $y$ are 4-adjacent, while $x \stackrel{0}{\sim} y$ if $x$ and $y$ are diagonally adjacent (i.e., 8-adjacent but not 4-adjacent). Consider the left configuration illustrated in Figure 2, where foreground and background pixels are marked 1 and 0 respectively:
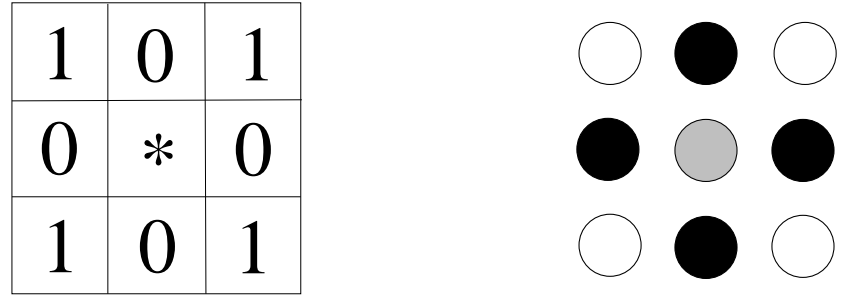

Fig. 2. A $3 \times 3$ binary image (left) and a corresponding $3 \times 3$ grey-scale image (right).

If the central pixel is a 1-pixel, then it is isolated in the figure, and the annular opening changes it into a 0-pixel. On the other hand if it is a 0-pixel, then it is isolated in the complement, and the annular closing changes it into a 1-pixel. Thus a combination of the behaviours of the annular opening and closing must interchange the values 0 and 1 in the central pixel, and so the resulting operator is neither increasing nor decreasing. Let us now see what happens when we try to extend this operator to grey-level images (it is known that any increasing operators for binary images extends to a "flat" operator for grey-level images $[1,8]$ ). The corresponding configuration is shown in Figure 2 (right), with white and black pixels in place of 1 - and 0-pixels; the central pixel has an intermediate grey value. Since all neighbours for $\stackrel{1}{\sim}$ of the central pixel are black, the annular opening changes its grey-level into black. On the other hand since all its neighbours for $\stackrel{0}{\sim}$ are white, the annular closing changes its grey-level into white.

The solution to this problem is to require that for every point $x$ there is some $y$ for which we have both $x \stackrel{0}{\sim} y$ and $x \stackrel{1}{\sim} y$; see Assumption 5.1. We show that in such a case we have an increasing operator, called annular operator, combining the behaviour of the annular opening and closing.

The idempotence of the annular operator is the most interesting problem that we study in this paper. We will derive conditions similar to the one in (1.6), on the two adjacency relations that guarantee that the annular operator is idempotent, in which case it will be called annular filter. 
In the case of translation-invariant operators, it will also be necessary, and we obtain an analogue of condition (1.7) involving two different symmetric structuring elements $A$ and $B$ :

$$
A \cap B \cap(A \oplus B) \neq \varnothing,
$$

or equivalently $0 \in(A \cap B) \oplus A \oplus B$. Then the operator $\psi$ given by

$$
\psi(X)=(X \cap(X \oplus A)) \cup(X \ominus B),
$$

will be a morphological filter removing isolated points from foreground and background, where the structuring elements $A$ and $B$ determine adjacency in foreground and background, respectively.

In the major part of this paper we will work in the general setting of an arbitrary space $E$ provided with two graph structures, one governing foreground adjacency and one for background adjacency.

Our paper is organized as follows: In Section 2 we will first recall some basic concepts from mathematical morphology. In Section 3 we summarize the results obtained in [3]. Although this is not really necessary, we have formulated our theory in terms of so-called switch pairs (Section 4). As an interesting side-result we obtain a new representation of an increasing translation invariant operator (Proposition 4.4). Then, in Section 5, we specialize to switch pairs based on adjacency, leading to a formal definition of an annular filter. In this section, our main result will be formulated. The translation invariant case is considered in Section 6. Finally, in Section 7, we apply some of the annular filters found before to a grey-scale and a binary image. Indeed, every increasing operator for binary images extends naturally into a flat operator for grey-level images, and annular filters can be extended in this way by using set structuring elements as flat structuring functions; their effect is to remove isolated light and dark spots in a grey-level image.

There are several aspects of annular filters that we do not consider here. First they could be generalized in the grey-level case by using grey-level structuring functions instead of flat ones. Indeed in [6], grey-level annular openings with grey-level structuring functions were analysed in a lattice-theoretical framework. The complex lattice-theoretical techniques necessary for such a study are very different from the graph-theoretical approach used here. Therefore we leave this question to a forthcoming mathematical paper [7].

The second question that we do not study here is whether an annular filter could be obtained as the composition of the corresponding annular opening and closing; a related question is whether the annular filter is a strong filter. Such questions have been often studied for filters built from usual morphological openings and closings, and solved through general arguments concerning increasing operators on complete lattices (see [9] and [2]). Again, such general argument do not fit into our binary graph-theoretic framework, so that these questions will also be dealt with in [7]. We will however translate these general results to the case of adjacency relations, and give the corresponding results without proof.

\section{Basic terminology}

In this section we recall some basic concepts from mathematical morphology which are used in the sequel of this paper. For more details the reader may refer to $[4,6,8,9]$. A comprehensive discussion can be found in [2].

Let $E$ be any set, e.g. the discrete space $\mathbb{Z}^{d}$ or the Euclidean space $\mathbb{R}^{d}$. We denote by $\mathcal{P}(E)$ the complete Boolean lattice of subsets of $E$. For a set $X \subseteq E$, we denote by $X^{\mathrm{c}}$ its 
complement. An operator $\psi: \mathcal{P}(E) \rightarrow \mathcal{P}(E)$ is increasing if $X \subseteq Y$ implies $\psi(X) \subseteq \psi(Y)$, for $X, Y \in \mathcal{P}(E)$. The dual or negation of $\psi$ is given by

$$
\psi^{*}(X)=\left(\psi\left(X^{\mathrm{c}}\right)\right)^{\mathrm{c}}
$$

The identity operator, mapping every set onto itself, is denoted by id.

2.1. Definition. An operator $\psi: \mathcal{P}(E) \rightarrow \mathcal{P}(E)$ is

(a) self-dual if $\psi^{*}=\psi$;

(b) a dilation if $\psi\left(\bigcup_{i \in I} X_{i}\right)=\bigcup_{i \in I} \psi\left(X_{i}\right)$, for $\left\{X_{i} \mid i \in I\right\} \subseteq \mathcal{P}(E)$;

(c) an erosion if $\psi\left(\bigcap_{i \in I} X_{i}\right)=\bigcap_{i \in I} \psi\left(X_{i}\right)$, for $\left\{X_{i} \mid i \in I\right\} \subseteq \mathcal{P}(E)$;

(d) idempotent if $\psi^{2}=\psi$;

(e) a filter if $\psi$ is increasing and idempotent;

(f) an opening if $\psi$ is a filter and satisfies $\psi(X) \subseteq X$ for every $X \in \mathcal{P}(E)$ (i.e., $\psi$ is antiextensive);

(g) a closing if $\psi$ is a filter and satisfies $X \subseteq \psi(X)$ for every $X \in \mathcal{P}(E)$ (i.e., $\psi$ is extensive).

2.2. Definition. Let $\varepsilon, \delta$ be operators on $\mathcal{P}(E)$. We say that $(\varepsilon, \delta)$ is an adjunction if

$$
\delta(X) \subseteq Y \Longleftrightarrow X \subseteq \varepsilon(Y)
$$

for $X, Y \in \mathcal{P}(E)$.

If $(\varepsilon, \delta)$ is an adjunction, then $\varepsilon$ is an erosion and $\delta$ a dilation. Furthermore, $\delta \varepsilon$ is an opening and $\varepsilon \delta$ a closing. To every erosion $\varepsilon$ there corresponds a unique dilation $\delta$, called the adjoint of $\varepsilon$, such that $(\varepsilon, \delta)$ forms an adjunction. The converse also holds.

If $(E,+)$ is an abelian group with unit element 0 (henceforth called the origin of $E$ ) then we say that $\psi$ is translation invariant if $\psi\left(X_{h}\right)=[\psi(X)]_{h}$ for $X \subseteq E$ and $h \in E$. Here $X_{h}=\{x+h \mid x \in X\}$. Every translation invariant dilation is a Minkowski addition:

$$
\delta(X)=X \oplus A=\bigcup_{a \in A} X_{a}
$$

The set $A \subseteq E$ is usually referred to as the structuring element. The adjoint erosion is the Minkowski subtraction

$$
\varepsilon(X)=X \ominus A=\bigcap_{a \in A} X_{-a} .
$$

The dilation $\delta$ is called symmetric if $x \in \delta(\{y\})$ iff $y \in \delta(\{x\})$. The translation invariant dilation $\delta(X)=X \oplus A$ is symmetric iff its structuring element $A$ is symmetric: $A=-A$.

There is a simple but important result due to Matheron [5] which says that every increasing, translation invariant operator can be decomposed in terms of Minkowski additions and subtractions. Define the kernel $\mathcal{V}(\psi)$ of an operator $\psi$ as

$$
\mathcal{V}(\psi)=\{A \subseteq E \mid 0 \in \psi(A)\}
$$

If $\psi$ is increasing and translation invariant, then

$$
\psi(X)=\bigcup_{A \in \mathcal{V}(\psi)} X \ominus A=\bigcap_{A \in \mathcal{V}\left(\psi^{*}\right)} X \oplus \check{A}
$$

where $\check{A}=-A$. 


\section{Switch operators and self-dual operators}

In this section we briefly recall some basic results about switch operators and self-dual operators on $\mathcal{P}(E)$ as developed in [3].

Denote, for a self-dual operator $\psi$, by $\sigma(X)$ the points which lie in $X$ but not in $\psi(X)$ :

$$
\sigma(X)=X \backslash \psi(X)=X \cap(\psi(X))^{\mathrm{c}}=X \cap \psi\left(X^{\mathrm{c}}\right) .
$$

Then $\sigma\left(X^{\mathrm{c}}\right)$ contains all points in $X^{\mathrm{c}}$ contained in $\psi(X)$. Therefore, $\psi$ is given by

$$
\psi(X)=(X \backslash \sigma(X)) \cup \sigma\left(X^{\mathrm{c}}\right)
$$

Let $\left(\mathcal{O}_{a e}, \leq\right)$ be the complete lattice of anti-extensive operators on $\mathcal{P}(E)$ with the usual ordering, and let $\left(\mathcal{O}_{s d}, \preccurlyeq\right)$ be the complete lattice of self-dual operators ordered by activity. An operator $\psi^{\prime}$ is more active than $\psi$ if $\psi^{\prime}$ affects more points than $\psi$ :

$$
\psi \preccurlyeq \psi^{\prime} \Longleftrightarrow \mathrm{id} \wedge \psi^{\prime} \leq \mathrm{id} \wedge \psi \text { and } \text { id } \vee \psi^{\prime} \geq \mathrm{id} \vee \psi
$$

see [9] or [2]. In [3] it is shown that relations (3.1)-(3.2) yield a lattice isomorphism between $\left(\mathcal{O}_{a e}, \leq\right)$ and $\left(\mathcal{O}_{s d}, \preccurlyeq\right)$. The intuitive meaning of this result is clear: let $\psi, \psi^{\prime} \in \mathcal{O}_{s d}$ correspond to $\sigma, \sigma^{\prime} \in \mathcal{O}_{a e}$; then the fact that $\psi^{\prime}$ is more active than $\psi$ means that for every $X \subseteq E, \psi^{\prime}$ removes more points from $X$ and adds to $X$ more points from $X^{c}$, in other words $\sigma(X) \subseteq \sigma^{\prime}(X)$ and $\sigma\left(X^{c}\right) \subseteq \sigma^{\prime}\left(X^{c}\right)$, that is $\sigma \leq \sigma^{\prime}$.

The self-dual operator $\psi$ given by (3.2) is increasing if and only if the anti-extensive operator $\sigma$ satisfies the two following conditions [3]:

(i) $X \cap \sigma(Y) \subseteq \sigma(X)$ if $X \subseteq Y$;

(ii) $\sigma\left(X^{\mathrm{c}}\right) \cap \sigma(Y)=\varnothing$ if $X \subseteq Y$.

If $(i)-(i i)$ hold, then $\sigma$ is called switch operator. The adjective 'switch' is used to indicate that $\sigma(X)$ and $\sigma\left(X^{\mathrm{c}}\right)$ are the points in $X$ and $X^{\mathrm{c}}$, respectively, which switch value from 1 to 0 and from 0 to 1 , respectively.

Consider the following example. Let $\psi$ be the operator on $\mathcal{P}\left(\mathbb{Z}^{2}\right)$ which translates a set by one pixel to the right. Evidently, $\psi$ is increasing and self-dual. Here $\sigma(X)$ is the left border of $X$, that is the set of pixels of $X$ whose left neighbour lies in $X^{c}$; indeed, $\psi$ removes from $X$ its left border $\sigma(X)$ and adds to it $\sigma\left(X^{c}\right)$, the left border of $X^{c}$. It is easily checked that $\sigma$ verifies conditions $(i)$ and $(i i)$.

In the remainder of this section we consider operators based upon the notion of adjacency. Let $\sim$ be a symmetric binary relation on the set $E$. We say that $x$ and $y$ are adjacent if $x \sim y$. $\operatorname{By} \operatorname{adj}(x)$ we denote the set of points which are adjacent to $x$ :

$$
\operatorname{adj}(x)=\{y \in E \mid x \sim y\}
$$

We assume that $\operatorname{adj}(x) \neq \varnothing$ for every $x \in E$. In [3] it is shown that the operator

$$
\sigma(X)=\{h \in X \mid \operatorname{adj}(h) \cap X=\varnothing\}
$$

is a switch operator. The operator $\psi$ satisfies:

- $h \in \psi(X)$ iff $(i) \operatorname{adj}(h) \subseteq X$ or $(i i) h \in X$ and there is $x \in X$ such that $h \sim x$.

- $h \notin \psi(X)$ iff $(i) \operatorname{adj}(h) \subseteq X^{\mathrm{c}}$ or $(i i) h \in X^{\mathrm{c}}$ and there is $x \in X^{\mathrm{c}}$ such that $h \sim x$. 
The operator $\psi$ can be written as

$$
\psi=(\mathrm{id} \wedge \delta) \vee \varepsilon=(\mathrm{id} \vee \varepsilon) \wedge \delta
$$

Here $\delta$ is the dilation given by

$$
\delta(X)=\{h \in E \mid \operatorname{adj}(h) \cap X \neq \varnothing\},
$$

and $\varepsilon$ is the adjoint erosion given by

$$
\varepsilon(X)=\{h \in E \mid \operatorname{adj}(h) \subseteq X\} .
$$

Moreover, $\varepsilon$ is the negative of $\delta$, i.e., $\varepsilon=\delta^{*}$, and

$$
\varepsilon \leq \delta
$$

This means in particular that $\psi$ is the centre operator of $\varepsilon$ and $\delta$; see [9] or [3].

The example mentioned above, where $\psi$ translates a set one pixel to the right, does not derive from a symmetric adjacency relation; the relation $x \sim y$ would be that $y$ is the left neighbour of $x$, which is not symmetric.

A question addressed in [3] concerns the idempotence of $\psi$. It was found that the socalled "adjacency triple condition" plays a key role here. Three points $x, y, z \in E$ are called an adjacency triple if $x \sim y \sim z \sim x$, and we say that $x, y, z$ are triple points. Note that $x, y, z$ need not be different.

3.1. Proposition. The operator $\psi$ in (3.4) is idempotent if and only if every point in $E$ is a triple point.

Proof. The 'if'-part has been proved in [3]. Here we prove the 'only if'-part.

Suppose that $h$ is not a triple point, and put $X=\operatorname{adj}(h)$. Note that $h \notin X$, for otherwise $h$ would belong to the adjacency triple $h, h, h$. It is obvious that $h \in \psi(X)$. Assume that $X \cap \psi(X) \neq \varnothing$, and let $x \in X \cap \psi(X)$. Then there must exist $y \in X$ such that $x \sim y$. But this means $h \sim x \sim y \sim h$, that is, $h, x, y$ is an adjacency triple, a contradiction. Therefore, $X \cap \psi(X)=\operatorname{adj}(h) \cap \psi(X)=\varnothing$, and as $h \in \psi(X)$, (3.3) gives that $h \in \sigma(\psi(X))$. This yields that $h \notin \psi^{2}(X)$. However, this means that $\psi$ is not idempotent, a contradiction.

Consider $E=\mathbb{Z}^{2}$ and assume that the adjacency relation is translation invariant: $x \sim y$ iff $x+h \sim y+h$ for all $x, y, h$. Then $\operatorname{adj}(h)=A_{h}$, where $A \subseteq \mathbb{Z}^{2}$ is a symmetric structuring element. In this case the adjacency triple condition amounts to

$$
0 \in A \oplus A \oplus A .
$$

An equivalent formulation is: $A \cap(A \oplus A) \neq \varnothing$. The corresponding self-dual idempotent operator is given by

$$
\psi(X)=(X \cap(X \oplus A)) \cup(X \ominus A) .
$$

Condition (3.7) is satisfied, for example, if

$$
A=\left[\begin{array}{lll}
\bullet & \bullet & \bullet \\
\bullet & \vdots & \bullet \\
\bullet & \bullet & \bullet
\end{array} \quad \text { or } \quad A=\left[\begin{array}{lllll}
\cdot & \bullet & \bullet & \bullet & \cdot \\
\bullet & \bullet & \ddots & \bullet & \bullet \\
\cdot & \cdot & \bullet & \cdot & \cdot
\end{array}\right.\right.
$$

The resulting operator $\psi$ is called annular filter. It removes isolated points in foreground and background, where 'isolated' has to be interpreted in terms of the adjacency relation induced by $A$. 


\section{Switch pairs}

The results summarized in the previous section can be generalized to the case where we consider different adjacencies for foreground and background. As a first step, we generalize the concept of a switch operator.

An easy computation shows that every operator $\psi$ on $\mathcal{P}(E)$ can be represented as

$$
\psi(X)=\left(X \backslash \sigma_{1}(X)\right) \cup \sigma_{0}\left(X^{\mathrm{c}}\right)
$$

where the anti-extensive operators $\sigma_{0}, \sigma_{1}$ are given by

$$
\sigma_{0}(X)=X \cap \psi\left(X^{\mathrm{c}}\right), \quad \sigma_{1}(X)=X \cap \psi(X)^{\mathrm{c}} .
$$

$\sigma_{1}(X)$ consists of all points in $X$ which are removed by $\psi$, and dually, $\sigma_{0}\left(X^{\mathrm{c}}\right)$ consists of all points in $X^{\mathrm{c}}$ which are added to $X$ by $\psi$. We reformulate these definitions in abstract terms.

Let, for $s=0,1$, the mappings $\Sigma_{s}: \mathcal{O} \rightarrow \mathcal{O}_{a e}$ be given by

$$
\Sigma_{s}(\psi)=\sigma_{s}
$$

That is,

$$
\Sigma_{0}(\psi)=\mathrm{id} \wedge \psi \nu, \quad \Sigma_{1}(\psi)=\mathrm{id} \wedge \nu \psi,
$$

where $\nu$ is the negation (complementation) operator $\nu(X)=X^{\mathrm{c}}$. Let $\Sigma: \mathcal{O} \rightarrow \mathcal{O}_{a e} \times \mathcal{O}_{a e}$ be given by $\Sigma(\psi)=\left(\Sigma_{0}(\psi), \Sigma_{1}(\psi)\right)$. Furthermore, we define $\Psi: \mathcal{O}_{a e} \times \mathcal{O}_{a e} \rightarrow \mathcal{O}$ by

$$
\Psi\left(\sigma_{0}, \sigma_{1}\right)=\left(\mathrm{id} \wedge \nu \sigma_{1}\right) \vee \sigma_{0} \nu
$$

The next result extends Propositions 3.3-3.4 in [3].

4.1. Proposition. The mapping $\Sigma:(\mathcal{O}, \preccurlyeq) \rightarrow\left(\mathcal{O}_{a e} \times \mathcal{O}_{a e}, \leq\right)$ is a complete lattice isomorphism with inverse $\Psi$.

Here the partial ordering on $\mathcal{O}_{a e} \times \mathcal{O}_{a e}$ is the pointwise ordering given by $\left(\sigma_{0}, \sigma_{1}\right) \leq\left(\sigma_{0}^{\prime}, \sigma_{1}^{\prime}\right)$ iff $\sigma_{0}(X) \subseteq \sigma_{0}^{\prime}(X)$ and $\sigma_{1}(X) \subseteq \sigma_{1}^{\prime}(X)$, for every $X \subseteq E$.

The proof of this statement is analogous to that of the corresponding results in [3]. Intuitively, given the operators $\psi, \psi^{\prime}$ corresponding to $\left(\sigma_{0}, \sigma_{1}\right)$ and $\left(\sigma_{0}^{\prime}, \sigma_{1}^{\prime}\right)$ respectively, it is clear that $\psi^{\prime}$ is more active than $\psi$ if and only if for every $X \subseteq E, \psi^{\prime}$ removes more points from $X$ and adds to $X$ more points from $X^{c}$, in other words $\sigma_{1}(X) \subseteq \sigma_{1}^{\prime}(X)$ and $\sigma_{0}\left(X^{c}\right) \subseteq \sigma_{0}^{\prime}\left(X^{c}\right)$, that is $\sigma_{1} \leq \sigma_{1}^{\prime}$ and $\sigma_{0} \leq \sigma_{0}^{\prime}$.

With respect to duality, the following results hold.

4.2. Proposition. $\Sigma(\psi)=\left(\sigma_{0}, \sigma_{1}\right)$ if and only if $\Sigma\left(\psi^{*}\right)=\left(\sigma_{1}, \sigma_{0}\right)$. In particular, $\psi$ is self-dual if and only if $\sigma_{0}=\sigma_{1}$.

In Section 2 we have given necessary and sufficient conditions on $\sigma$ for the increasingness of $\psi$. We generalize these conditions to the present framework. Note that the first condition consists of two parts, one for $s=0$ and one for $s=1$.

( $\left.\mathrm{I}_{s}\right) X \cap \sigma_{s}(Y) \subseteq \sigma_{s}(X)$, if $X \subseteq Y$;

(II) $\sigma_{0}\left(X^{\mathrm{c}}\right) \cap \sigma_{1}(Y)=\varnothing$, if $X \subseteq Y$.

An equivalent formulation of (II) is:

$\sigma_{0}(X) \cap \sigma_{1}(Y)=\varnothing$ if $X \cup Y=E$. 
4.3. Proposition. Let $\sigma_{0}, \sigma_{1}$ be anti-extensive operators on $\mathcal{P}(E)$. The operator $\psi=\Psi\left(\sigma_{0}, \sigma_{1}\right)$ is increasing if and only if $\left(\mathrm{I}_{0}\right),\left(\mathrm{I}_{1}\right)$, (II) hold.

Proof. It is easy to see that these conditions are necessary. To prove sufficiency, assume that all of them hold and that $X \subseteq Y$; we must show that $\psi(X) \subseteq \psi(Y)$. Assume $h \in \psi(X)$; we distinguish three cases.

1. $h \in X$ : thus $h \notin \sigma_{1}(X)$. Suppose that $h \in \sigma_{1}(Y)$; then, by $\left(\mathrm{I}_{1}\right), h \in \sigma_{1}(X)$, a contradiction. Therefore, $h \notin \sigma_{1}(Y)$, and we get that $h \in Y \backslash \sigma_{1}(Y) \subseteq \psi(Y)$.

2. $h \notin X$ and $h \in Y$ : as $h \in \psi(X)$, we conclude that $h \in \sigma_{0}\left(X^{\mathrm{c}}\right)$. From (II) we derive that $h \notin \sigma_{1}(Y)$, and thus $h \in \psi(Y)$.

3. $h \notin Y$ : thus $h \in \psi(X) \backslash X=\sigma_{0}\left(X^{\mathrm{c}}\right)$. Applying $\left(\mathrm{I}_{0}\right)$ to $Y^{\mathrm{c}}$ and $X^{\mathrm{c}}$, we find that $Y^{\mathrm{c}} \cap \sigma_{0}\left(X^{\mathrm{c}}\right) \subseteq \sigma_{0}\left(Y^{\mathrm{c}}\right)$. This yields that $h \in \sigma_{0}\left(Y^{\mathrm{c}}\right)$, and therefore $h \in \psi(Y)$.

We say that $\left(\sigma_{0}, \sigma_{1}\right)$ is a switch pair if conditions $\left(\mathrm{I}_{0}\right),\left(\mathrm{I}_{1}\right)$, (II) hold. From Proposition 4.2 we derive that $\left(\sigma_{0}, \sigma_{1}\right)$ is a switch pair if and only if $\left(\sigma_{1}, \sigma_{0}\right)$ is one. Furthermore, $(\sigma, \sigma)$ is a switch pair if and only if $\sigma$ is a switch operator.

Consider the following example. Let $\psi$ be the operator on $\mathcal{P}\left(\mathbb{Z}^{2}\right)$ which transforms a set into the union of its two translates by one pixel to the left and to the right respectively; clearly $\psi$ is increasing. Here $\sigma_{0}(X)$ is the union of the left and right borders of $X$, while $\sigma_{1}(X)$ is the intersection of the left and right borders of $X$. It is easily checked that $\psi$ satisfies $\left(\mathrm{I}_{0}\right),\left(\mathrm{I}_{1}\right)$, and (II).

In the remainder of this section we restrict ourselves to translation invariant operators. Let $\psi$ be an increasing translation invariant operator on $\mathcal{P}(E)$. Using the kernel representation (2.1) and (4.2) we have

$$
\begin{aligned}
\sigma_{0}(X) & =X \cap \psi\left(X^{\mathrm{c}}\right)=X \cap \bigcup_{A \in \mathcal{V}(\psi)} X^{\mathrm{c}} \ominus A \\
& =X \cap \bigcup_{A_{0} \in \mathcal{A}_{0}} X^{\mathrm{c}} \ominus A_{0},
\end{aligned}
$$

where $\mathcal{A}_{0}=\{A \in \mathcal{V}(\psi) \mid 0 \notin A\}$. Here we have used that $X \cap\left(X^{\mathrm{c}} \ominus A\right)=\varnothing$ if $0 \in A$. A similar computation shows that

$$
\sigma_{1}(X)=X \cap \bigcup_{A_{1} \in \mathcal{A}_{1}} X^{\mathrm{c}} \ominus A_{1},
$$

where $\mathcal{A}_{1}=\left\{A \in \mathcal{V}\left(\psi^{*}\right) \mid 0 \notin A\right\}$.

Suppose that $A_{0} \in \mathcal{A}_{0}$ and $A_{1} \in \mathcal{A}_{1}$, then $0 \in \psi\left(A_{0}\right)$ and $0 \in \psi^{*}\left(A_{1}\right)$, i.e., $0 \notin \psi\left(A_{1}^{\mathrm{c}}\right)$. Suppose that $A_{0} \cap A_{1}=\varnothing$, then $A_{0} \subseteq A_{1}^{\mathrm{c}}$, and by the increasingness of $\psi$ we get that $0 \in \psi\left(A_{1}^{\mathrm{c}}\right)$, a contradiction. Therefore, we may conclude that

$$
A_{0} \cap A_{1} \neq \varnothing, \quad \text { for } A_{0} \in \mathcal{A}_{0}, A_{1} \in \mathcal{A}_{1} \text {. }
$$

The operator $\psi$ is given by

$$
\begin{aligned}
\psi(X) & =\left(X \backslash \sigma_{1}(X)\right) \cup \sigma_{0}\left(X^{\mathrm{c}}\right) \\
& =X \cap\left(X \cap \bigcup_{A_{1} \in \mathcal{A}_{1}} X^{\mathrm{c}} \ominus A_{1}\right)^{\mathrm{c}} \cup\left(X^{\mathrm{c}} \cap \bigcup_{A_{0} \in \mathcal{A}_{0}} X \ominus A_{0}\right) \\
& =X \cap\left(X^{\mathrm{c}} \cup \bigcap_{A_{1} \in \mathcal{A}_{1}} X \oplus \check{A}_{1}\right) \cup\left(X^{\mathrm{c}} \cap \bigcup_{A_{0} \in \mathcal{A}_{0}} X \ominus A_{0}\right) \\
& =\left(X \cap \bigcap_{A_{1} \in \mathcal{A}_{1}} X \oplus \check{A}_{1}\right) \cup\left(X^{\mathrm{c}} \cap \bigcup_{A_{0} \in \mathcal{A}_{0}} X \ominus A_{0}\right) .
\end{aligned}
$$


As $A_{0} \cap A_{1} \neq \varnothing$ for $A_{0} \in \mathcal{A}_{0}$ and $A_{1} \in \mathcal{A}_{1}$, it follows that $X \ominus A_{0} \subseteq X \oplus \check{A}_{1}$. Therefore $\bigcup_{A_{0} \in \mathcal{A}_{0}} X \ominus A_{0} \subseteq \bigcap_{A_{1} \in \mathcal{A}_{1}} X \oplus \check{A}_{1}$, and thus we get

$$
\begin{aligned}
\psi(X) & =\left(X \cap \bigcap_{A_{1} \in \mathcal{A}_{1}} X \oplus \check{A}_{1}\right) \cup\left(X \cap \bigcup_{A_{0} \in \mathcal{A}_{0}} X \ominus A_{0}\right) \cup\left(X^{\mathrm{c}} \cap \bigcup_{A_{0} \in \mathcal{A}_{0}} X \ominus A_{0}\right) \\
& =\left(X \cap \bigcap_{A_{1} \in \mathcal{A}_{1}} X \oplus \check{A}_{1}\right) \cup \bigcup_{A_{0} \in \mathcal{A}_{0}} X \ominus A_{0},
\end{aligned}
$$

where we have used that

$$
\left(X \cap \bigcup_{A_{0} \in \mathcal{A}_{0}} X \ominus A_{0}\right) \cup\left(X^{\mathrm{c}} \cap \bigcup_{A_{0} \in \mathcal{A}_{0}} X \ominus A_{0}\right)=\bigcup_{A_{0} \in \mathcal{A}_{0}} X \ominus A_{0}
$$

We reformulate this result in terms of the centre operator.

4.4. Proposition. Let $\psi$ be an increasing translation invariant operator on $\mathcal{P}(E)$, and let

$$
\mathcal{A}_{0}=\{A \in \mathcal{V}(\psi) \mid 0 \notin A\}, \quad \mathcal{A}_{1}=\left\{A \in \mathcal{V}\left(\psi^{*}\right) \mid 0 \notin A\right\} .
$$

For every $A_{0} \in \mathcal{A}_{0}$ and $A_{1} \in \mathcal{A}_{1}$, we have

$$
A_{0} \cap A_{1} \neq \varnothing
$$

Define the increasing operators $\psi_{0}, \psi_{1}$ by

$$
\psi_{0}(X)=\bigcup_{A_{0} \in \mathcal{A}_{0}} X \ominus A_{0}, \quad \psi_{1}(X)=\bigcap_{A_{1} \in \mathcal{A}_{1}} X \oplus \check{A}_{1} .
$$

Then $\psi_{0} \leq \psi_{1}$ and $\psi$ is the centre of $\psi_{0}$ and $\psi_{1}$, i.e.,

$$
\begin{aligned}
\psi(X) & =\left(X \cap \bigcap_{A_{1} \in \mathcal{A}_{1}} X \oplus \check{A}_{1}\right) \cup \bigcup_{A_{0} \in \mathcal{A}_{0}} X \ominus A_{0} \\
& =\left(X \cup \bigcup_{A_{0} \in \mathcal{A}_{0}} X \ominus A_{0}\right) \cap \bigcap_{A_{1} \in \mathcal{A}_{1}} X \oplus \check{A}_{1} .
\end{aligned}
$$

Observe that $\mathcal{A}_{0}$ and $\mathcal{A}_{1}$ contain the patterns for which the origin is a switch point. To be precise: given a set $X$ with $0 \in X$, then $0 \notin \psi(X)$ iff there is some $A_{0} \in \mathcal{A}_{0}$ completely contained in $X^{\mathrm{c}}$; given a set $X$ with $0 \notin X$, then $0 \in \psi(X)$ iff there is some $A_{1} \in \mathcal{A}_{1}$ completely contained in $X$. The more patterns that are contained in $\mathcal{A}_{0}$ and $\mathcal{A}_{1}$ (but such that (4.5) holds), the more active is $\psi$. In practice, we will only use subsets of $\mathcal{A}_{0}$ and $\mathcal{A}_{1}$ defined in (4.4), since both sets contain infinitely many redundant patterns. For example, if $A \in \mathcal{A}_{0}$ and $A^{\prime}$ is such that $0 \notin A^{\prime}$ and $A \subseteq A^{\prime}$, then $A^{\prime} \in \mathcal{A}_{0}$ as well. However, it can be deleted from the representation in (4.7).

\section{Adjacency and annular filters}

A simple and intuitive way to construct switch pairs is by using the concept of adjacency; see also Section 3. The corresponding operator switches those points in $X$ and $X^{\mathrm{c}}$ which are isolated in the sense of an adjacency relation (or neighbour relation) to be specified. In this section we investigate switch pairs based on adjacency relations and we provide sufficient conditions for idempotence of the resulting operators. 
Consider two symmetric relations $\stackrel{0}{\sim}$ and $\stackrel{1}{\sim}$ on $E \times E$. We refer to these two relations as background and foreground adjacency, respectively. Define, for $s=0,1$ and $x \in E$ :

$$
\operatorname{adj}_{s}(x)=\{y \in E \mid x \stackrel{s}{\sim} y\}
$$

Throughout this section we assume that $\operatorname{adj}_{s}(x) \neq \varnothing$, for $s=0,1$ and $x \in E$. Let the antiextensive operator $\sigma_{s}$ be given by

$$
\sigma_{s}(X)=\left\{h \in X \mid X \cap \operatorname{adj}_{s}(h)=\varnothing\right\}
$$

Let $\psi$ be the operator given by (4.1); we see that:

- $h \in \psi(X)$ iff

(i) $h \in X^{\mathrm{c}}$ and $\operatorname{adj}_{0}(h) \subseteq X$ or

(ii) $h \in X$ and there is $x \in X$ such that $h \stackrel{1}{\sim} x$.

- $h \notin \psi(X)$ iff

(i) $h \in X$ and $\operatorname{adj}_{1}(h) \subseteq X^{\mathrm{c}}$ or

(ii) $h \in X^{\mathrm{c}}$ and there is $x \in X^{\mathrm{c}}$ such that $h \stackrel{0}{\sim} x$.

It is easy to verify that condition $\left(\mathrm{I}_{s}\right)$ introduced in the previous section is satisfied. For (II) to be satisfied we need an extra requirement.

5.1. Assumption. For every $x \in E$ there exists a $y \in E$ such that $x \stackrel{0}{\sim} y \stackrel{1}{\sim} x$.

5.2. Lemma. Let $\sigma_{0}, \sigma_{1}$ be given by (5.1). Condition (II) holds if Assumption 5.1 is satisfied. If no point in $E$ is background- or foreground-adjacent to itself, then the converse also holds.

Proof. Suppose that Assumption 5.1 holds. We must show that $\sigma_{0}\left(X^{\mathrm{c}}\right) \cap \sigma_{1}(Y)=\varnothing$ if $X \subseteq Y$. Assume that $x \in \sigma_{0}\left(X^{\mathrm{c}}\right) \cap \sigma_{1}(Y)$, then $\operatorname{adj}_{0}(x) \cap X^{\mathrm{c}}=\varnothing$ and $\operatorname{adj}_{1}(x) \cap Y=\varnothing$. Let $y$ be such that $x \stackrel{0}{\sim} y \stackrel{1}{\sim} x$, then $y \in X$ and $y \notin Y$, a contradiction.

Suppose (II) holds. Let $x \in E$ and assume that $\operatorname{adj}_{0}(x) \cap \operatorname{adj}_{1}(x)=\varnothing$. Put $X=\operatorname{adj}_{0}(x)$ and $Y=\left[\operatorname{adj}_{1}(x)\right]^{\mathrm{c}}$, then $X \subseteq Y$. If $x \not{\psi} x$ and If $x \not{\psi} x$, then $x \in \sigma_{0}\left(X^{\mathrm{c}}\right) \cap \sigma_{1}(Y)$, which contradicts (II).

Therefore, if Assumption 5.1 holds, the operator $\psi$ given by (4.1) is increasing.

For $s=0,1$ we define the adjunction $\left(\varepsilon_{s}, \delta_{s}\right)$ on $\mathcal{P}(E)$ by

$$
\begin{aligned}
& \delta_{s}(X)=\left\{h \in E \mid \operatorname{adj}_{s}(h) \cap X \neq \varnothing\right\} \\
& \varepsilon_{s}(X)=\left\{h \in E \mid \operatorname{adj}_{s}(h) \subseteq X\right\}
\end{aligned}
$$

From the fact that $\operatorname{adj}_{s}(h) \neq \varnothing$ we get immediately that

$$
\varepsilon_{s} \leq \delta_{s}
$$

Furthermore, by the symmetry of both adjacencies:

$$
\varepsilon_{s}=\delta_{s}^{*}
$$

5.3. Remark. The dilation $\delta_{s}$ is symmetric in the sense that $y \in \delta_{s}(\{x\})$ iff $x \in \delta_{s}(\{y\})$. Now it follows easily that id $\wedge \delta_{s}$ is an opening, a so-called annular opening. Dually, id $\vee \varepsilon_{s}$ is an annular closing. 
A straightforward computation shows that

$$
\sigma_{s}=\mathrm{id} \wedge \nu \delta_{s}=\mathrm{id} \wedge \varepsilon_{s} \nu
$$

Thus the operator $\psi=\Psi\left(\sigma_{0}, \sigma_{1}\right)$ is given by

$$
\begin{aligned}
\psi & =\left(\mathrm{id} \wedge \nu \sigma_{1}\right) \vee \sigma_{0} \nu=\left(\mathrm{id} \wedge\left(\nu \vee \delta_{1}\right)\right) \vee\left(\nu \wedge \varepsilon_{0}\right) \\
& =\left(\mathrm{id} \wedge \delta_{1}\right) \vee\left(\nu \wedge \varepsilon_{0}\right)
\end{aligned}
$$

5.4. Proposition. If Assumption 5.1 holds then $\varepsilon_{0} \leq \delta_{1}$ and $\varepsilon_{1} \leq \delta_{0}$, and $\psi$ is the centre operator of $\varepsilon_{0}$ and $\delta_{1}$, i.e.

$$
\psi=\left(\mathrm{id} \wedge \delta_{1}\right) \vee \varepsilon_{0}=\left(\mathrm{id} \vee \varepsilon_{0}\right) \wedge \delta_{1} .
$$

Proof. It is easy to see that $\varepsilon_{0} \leq \delta_{1}$ and $\varepsilon_{1} \leq \delta_{0}$ if Assumption 5.1 holds. Now id $\wedge \delta_{1} \geq \mathrm{id} \wedge \varepsilon_{0}$ and the expression in (5.7) equals

$$
\begin{aligned}
\psi & =\left(\mathrm{id} \wedge \delta_{1}\right) \vee\left(\nu \wedge \varepsilon_{0}\right) \vee\left(\mathrm{id} \wedge \varepsilon_{0}\right) \\
& =\left(\mathrm{id} \wedge \delta_{1}\right) \vee\left[(\nu \vee \mathrm{id}) \wedge \varepsilon_{0}\right] \\
& =\left(\mathrm{id} \wedge \delta_{1}\right) \vee \varepsilon_{0} .
\end{aligned}
$$

This concludes the proof.

It is in fact easy to show that Assumption 5.1 holds iff $\varepsilon_{0} \leq \delta_{1}$ iff $\varepsilon_{1} \leq \delta_{0}$. It is also easy to see that

$$
\psi^{*}=\left(\mathrm{id} \wedge \delta_{0}\right) \vee \varepsilon_{1}=\left(\mathrm{id} \vee \varepsilon_{1}\right) \wedge \delta_{0} .
$$

In other words, $\psi^{*}$ is the operator obtained by interchanging the roles of foreground and background adjacency, as one could have expected.

Now we address the problem concerning the idempotence of $\psi$. For future reference we make the following two dual observations.

$\left(\mathrm{O}_{1}\right)$ Given $x \in X$, we have $x \in \psi(X)$ iff there exists a point $y \in X$ such that $x \stackrel{1}{\sim} y$. In this case, $x, y \in \psi^{n}(X)$ for $n \geq 0$. We say that the pair $x, y$ forms a stable 1-configuration.

$\left(\mathrm{O}_{0}\right)$ Given $x \notin X$, we have $x \notin \psi(X)$ iff there exists a point $y \notin X$ such that $x \stackrel{0}{\sim} y$. In this case, $x, y \notin \psi^{n}(X)$ for $n \geq 0$. We say that the pair $x, y$ forms a stable 0 -configuration.

We need a generalization of the adjacency triple condition stated in Section 3.

5.5. Definition. A point $x \in E$ is called a 0 -triple point if there exist $y, z \in E$ such that

$$
x \stackrel{0}{\sim} z \stackrel{1}{\sim} y \stackrel{0}{\sim} x \stackrel{1}{\sim} y
$$

Dually, a point $x$ is called a 1-triple point if there exist $y, z \in E$ such that

$$
x \stackrel{1}{\sim} z \stackrel{0}{\sim} y \stackrel{1}{\sim} x \stackrel{0}{\sim} y
$$

Finally, it is called a $0 / 1$-triple point if there exist $y, z \in E$ such that

$$
y \stackrel{0}{\sim} x \stackrel{1}{\sim} z \stackrel{0}{\sim} y \stackrel{1}{\sim} z
$$

In Figure 3 we illustrate these notions by means of adjacency graphs.

Let $X \subseteq E$. We introduce the following symbolic notation. If $x \in E$ and $n$ a nonnegative integer, we write $x(n)=1$ if $x \in \psi^{n}(X)$ and 0 otherwise. We call $x(\cdot)$ the trajectory of $x$ with respect to $X$. 


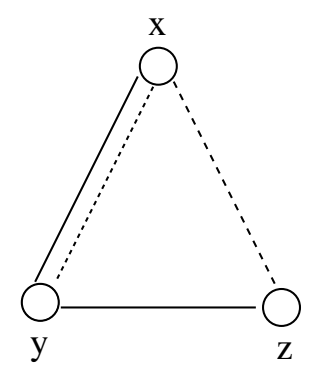

Fig. 3. A solid line denotes foreground adjacency, a dashed line background adjacency. Here $x$ is a 0 -triple point, $y$ a 1-triple point, and $z$ a $0 / 1$-triple point.

\subsection{Lemma.}

(a) If $x$ is a 0-triple point, then $x(1) \leq x(2)=x(3)=\cdots$.

(b) If $x$ is a 1-triple point, then $x(1) \geq x(2)=x(3)=\cdots$.

Proof. We prove (a); then (b) follows by duality. We consider two cases: $(i) x(1)=1$, and (ii) $x(1)=0$.

(i) Assume $x(1)=1$. If $x(0)=1$, then, by observation $\left(\mathrm{O}_{1}\right)$, it follows that $x(n)=1$ for $n \geq 0$. Suppose now that $x(0)=0$, then $x \in \psi(X) \backslash X$, hence $\operatorname{adj}_{0}(x) \subseteq X$. Let $x, y, z$ be as in Figure 3; then $y(0)=z(0)=1$. Since $y, z$ are 1-adjacent, observation $\left(\mathrm{O}_{1}\right)$ implies that $y(n)=z(n)=1$ for $n \geq 0$. Thus $x(1)=y(1)=1$ and, again by $\left(\mathrm{O}_{1}\right), x(n)=1$ for $n \geq 1$.

(ii) Let $x(1)=0$. If $x(2)=0$ then, by $\left(\mathrm{O}_{0}\right), x(n)=0$ for $n \geq 1$. Assume $x(2)=1$ and put $x^{\prime}(n)=x(n+1)$. Observe that $x^{\prime}(\cdot)$ is the trajectory of $x$ with respect to $\psi(X)$. Now $x^{\prime}(1)=1$ and $x^{\prime}(0)=0$. The same arguments as in $(i)$ yield $x^{\prime}(n)=1$ for $n \geq 1$, hence $x(n)=1$ for $n \geq 2$.

From this lemma one can easily deduce the following two results.

5.7. Corollary. If every point in $E$ is a 0-triple point (resp. 1-triple point) then $\psi^{2} \geq \psi$ (resp. $\left.\psi^{2} \leq \psi\right)$. In particular, if every point in $E$ is both a 1-triple point and a 0-triple point, then $\psi$ is a filter.

5.8. Corollary. If every point in $E$ is a 1-triple point or a 0 -triple point, then $\psi^{3}=\psi^{2}$.

The examples in Figure 4 show that idempotence cannot be established if we merely assume that every point is either a 0 -triple point or a 1-triple point. In both examples two iterations are required before the configuration becomes stable, in other words: $\psi^{3}=\psi^{2}$.

We can also prove the following result.

\subsection{Proposition.}

(a) If $\psi^{2} \geq \psi$, then every point $x \in E$ is a 0-triple point or a 0/1-triple point.

(b) If $\psi^{2} \leq \psi$, then every point $x \in E$ is a 1-triple point or a 0/1-triple point.

Proof. We prove (a); then (b) follows by duality. Let $x \in E$ and define $X=\operatorname{adj}_{0}(x)$. If $x \in X$ then we get, in combination with Assumption 5.1, that $x$ is a 0 -triple point. We assume henceforth that $x \notin X$. It follows immediately that $x \in \psi(X)$ and, as $\psi^{2} \geq \psi$, we have $x \in \psi^{2}(X)$. Therefore, $x \notin \sigma_{1}(\psi(X))$, i.e., $\operatorname{adj}_{1}(x) \cap \psi(X) \neq \varnothing$. Take $y \in \operatorname{adj}_{1}(x) \cap \psi(X)$. There are two possibilities: $y \in X$ and $y \notin X$. 

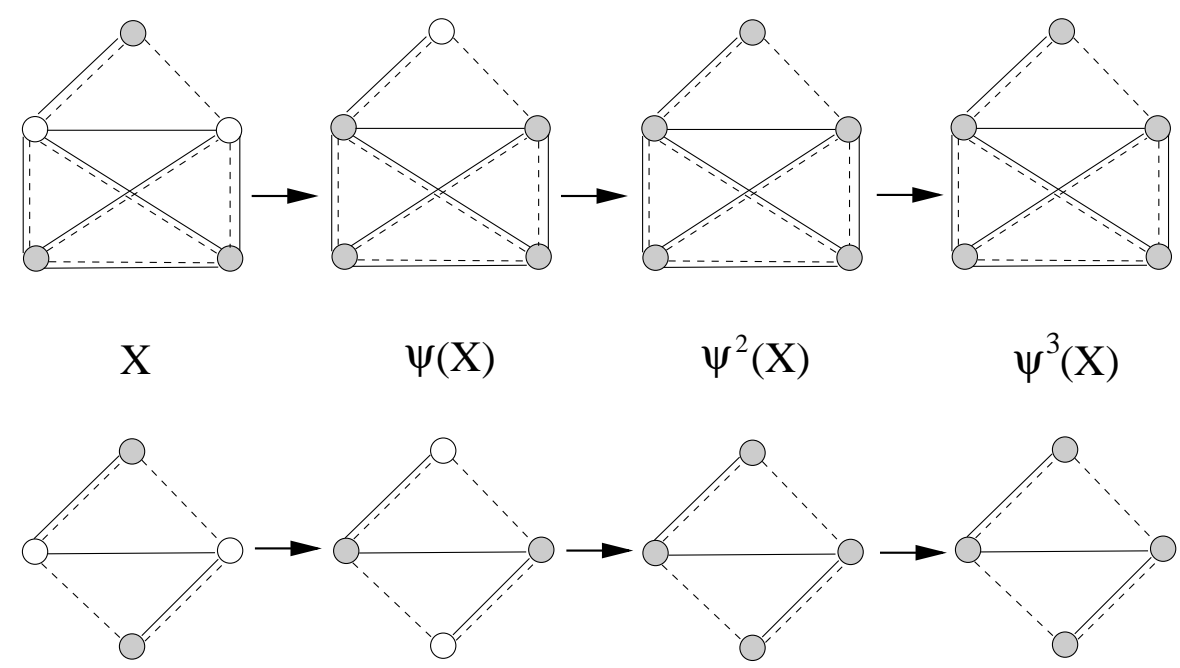

Fig. 4. Two illustrations of $\psi$. A solid line denotes foreground adjacency, a dashed line background adjacency. The set $X$ consists of the grey points. In the first example, $E$ contains five points, each of which is a 0 -triple point. In this case $\psi(X) \varsubsetneqq \psi^{2}(X)=\psi^{3}(X)$. In the second example, $E$ contains four points, two of which are 1-triple points and two of which are 0 -triple points. We have $\psi(X) \neq \psi^{2}(X)=\psi^{3}(X)$.

' $y \in X$ ': thus $y \stackrel{0}{\sim} x \stackrel{1}{\sim} y$. Since $y \in \psi(X)$ we must have that $\operatorname{adj}_{1}(y) \cap X \neq \varnothing$, i.e., $\operatorname{adj}_{1}(y) \cap \operatorname{adj}_{0}(x) \neq \varnothing$. But then for $z \in \operatorname{adj}_{1}(y) \cap \operatorname{adj}_{0}(x)$ we have $x \stackrel{0}{\sim} z \stackrel{1}{\sim} y \stackrel{0}{\sim} x \stackrel{1}{\sim} y$ and $x$ is a 0 -triple point.

' $y \notin X '$ ' since $y \in \psi(X)$, we have $y \in \sigma_{0}\left(X^{\mathrm{c}}\right) . \operatorname{Thus}_{\operatorname{adj}_{0}}(y) \cap X^{\mathrm{c}}=\varnothing$, i.e., $\operatorname{adj}_{0}(y) \subseteq$ $X=\operatorname{adj}_{0}(x)$. By Assumption 5.1, there exists a $z$ with $z \stackrel{0}{\sim} y \stackrel{1}{\sim} z$. Then, also $z \stackrel{0}{\sim} x$, and we get $z \stackrel{0}{\sim} x \stackrel{1}{\sim} y \stackrel{0}{\sim} z \stackrel{1}{\sim} y$, thus $x$ is a $0 / 1$-triple point.

In [7] the following result is shown.

5.10. Proposition. Let Assumption 5.1 hold.

(a) Assume that $x \stackrel{0}{\sim} y \Rightarrow \exists z \in E: x \stackrel{0}{\sim} z \stackrel{1}{\sim} y$. Then $\psi=\left(\right.$ id $\left.\vee \varepsilon_{0}\right)\left(\right.$ id $\left.\wedge \delta_{1}\right)$ and $\psi$ is an inf-filter.

(b) Assume that $x \stackrel{1}{\sim} y \Rightarrow \exists z \in E: x \stackrel{1}{\sim} z \stackrel{0}{\sim} y$. Then $\psi=\left(\mathrm{id} \wedge \delta_{1}\right)\left(\mathrm{id} \vee \varepsilon_{0}\right)$ and $\psi$ is a sup-filter.

Observe that the assumption in (a) in combination with Assumption 5.1 implies that every point in $E$ is a 0 -triple point and a 1-triple point. Similarly for the assumption in (b).

\section{Translation invariant case}

Assume that $(E,+)$ is an abelian group, e.g. $\mathbb{Z}^{d}$ or $\mathbb{R}^{d}$, and that both adjacencies are translation invariant, i.e. $x \stackrel{s}{\sim} y$ if and only if $x+h \stackrel{s}{\sim} y+h$, for $x, y, h \in E$ and $s=0,1$. Thus there exist symmetric structuring elements $A, B \subseteq E$ such that

$$
\operatorname{adj}_{1}(h)=A_{h}, \quad \operatorname{adj}_{0}(h)=B_{h} .
$$

Assumption 5.1 means that

$$
A \cap B \neq \varnothing .
$$


Now the operator $\psi$ is given by

$$
\psi(X)=(X \cap(X \oplus A)) \cup(X \ominus B) .
$$

We point out that this is a special case of the expression in (4.7), with $\mathcal{A}_{0}$ and $\mathcal{A}_{1}$ both containing one structuring element.

Because of the translation-invariance of the adjacency relations, it is clear that either the 3 -point configuration of Figure 3 does not appear, in other words there are no 0-, 1-, or 0/1-triple points, or such a configuration appears everywhere, and so every point of $E$ is at the same time a 0 -, 1-, and 0/1-triple point. This holds if and only if

$$
A \cap B \cap(A \oplus B) \neq \varnothing .
$$

An equivalent formulation is $0 \in(A \cap B) \oplus A \oplus B$. Thus we find the following generalization of Theorem 4.7 in [3].

6.1. Proposition. Let $A, B$ be two symmetric structuring elements in $E$. The operator $\psi(X)=$ $(X \cap(X \oplus A)) \cup(X \ominus B)$ is a morphological filter if and only if (6.2) holds. It is self-dual if and only if $A=B$.

This result follows easily from Corollary 5.7 and Proposition 5.9.

Observe that $\psi(X)=X \cup(X \ominus B)$ if $0 \in A$, and that $\psi(X)=X \cap(X \oplus A)$ if $0 \in B$.

We present some examples; in all examples one may interchange $A$ and $B$.
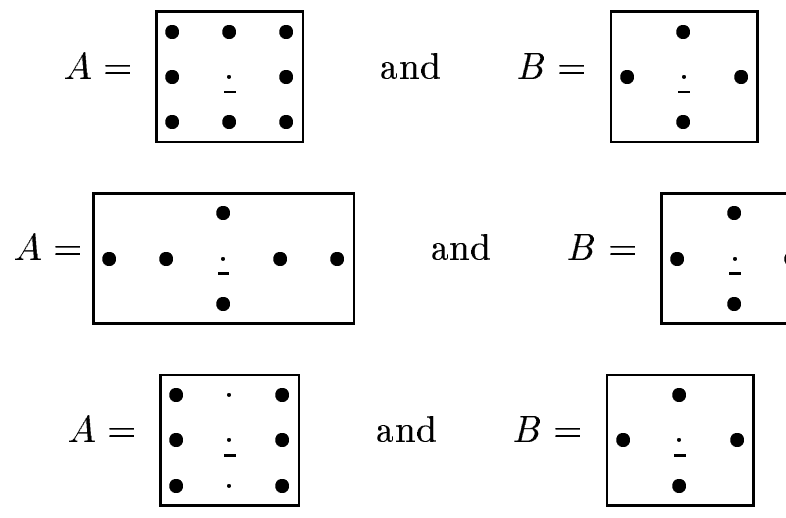

The assumptions in (a) and (b) of Proposition 5.10 reduce to, respectively:

$$
B \subseteq A \oplus B \text { and } A \subseteq A \oplus B
$$

Combination of both conditions lead to the following result.

6.2. Corollary. Let $A, B$ be two symmetric structuring elements which satisfy $A \cap B \neq \varnothing$ and

$$
A \cup B \subseteq A \oplus B,
$$

then the operator $\psi=\left(\mathrm{id} \wedge \delta_{A}\right) \vee \varepsilon_{B}$ is a strong filter and can be written as the composition in either order of the annular opening id $\vee \varepsilon_{B}$ and the annular closing id $\wedge \delta_{A}$, i.e.,

$$
\psi=\left(\mathrm{id} \wedge \delta_{A}\right)\left(\mathrm{id} \vee \varepsilon_{B}\right)=\left(\mathrm{id} \vee \varepsilon_{B}\right)\left(\mathrm{id} \wedge \delta_{A}\right)
$$

Observe that (6.2) is automatically satisfied if $A \cap B \neq \varnothing$ and (6.3) holds. It is easy to verify that (6.3) is satisfied for the first and the third example above, but not for the second. 
In Proposition 5.8 it is stated that $\psi^{3}=\psi^{2}$ if every point in $E$ is a 0 - or a 1-triple point. But, as we observed above, in the translation invariant case this automatically implies that every point is of both types. This becomes different if we also relax the assumption that $\psi$ is translation invariant.

In the following we consider only the special case where $E=\mathbb{Z}^{2}$. We say that an operator $\xi$ on $\mathcal{P}\left(\mathbb{Z}^{2}\right)$ is even-translation invariant if

$$
\xi\left(X_{h}\right)=[\xi(X)]_{h}
$$

for every $X \subseteq \mathbb{Z}^{2}$ and for every even vector $h \in \mathbb{Z}^{2}$; a vector $h=\left(h_{1}, h_{2}\right)$ is called even if $h_{1}+h_{2}$ is an even integer, otherwise it is called odd. We write $\mathbb{Z}^{2}=\mathbb{Z}_{e}^{2} \cup \mathbb{Z}_{o}^{2}$, where $\mathbb{Z}_{e}^{2}$ contains the even vectors and $\mathbb{Z}_{o}^{2}$ the odd vectors.

In this case, the shape of the set $\operatorname{adj}_{s}(h)$ is different for odd and even vectors. Assume

$$
\operatorname{adj}_{1}(h)=\left\{\begin{array}{ll}
\left(A_{e}\right)_{h}, & \text { if } h \text { is even } \\
\left(A_{o}\right)_{h}, & \text { if } h \text { is odd }
\end{array} \text { and } \operatorname{adj}_{0}(h)= \begin{cases}\left(B_{e}\right)_{h}, & \text { if } h \text { is even } \\
\left(B_{o}\right)_{h}, & \text { if } h \text { is odd }\end{cases}\right.
$$

Here $A_{e}, A_{o}, B_{e}, B_{o} \subseteq \mathbb{Z}^{2}$. The assumption that the adjacency relations are symmetric lead to the following conditions:

- $A_{e} \cap \mathbb{Z}_{e}^{2}$ is symmetric;

- $A_{o} \cap \mathbb{Z}_{e}^{2}$ is symmetric;

- $A_{e} \cap \mathbb{Z}_{o}^{2}=-\left(A_{o} \cap \mathbb{Z}_{o}^{2}\right)$.

For $B_{e}$ and $B_{o}$ we find similar symmetry conditions. Furthermore, Assumption 5.1 requires that - $A_{e} \cap B_{e} \neq \varnothing$ and $A_{o} \cap B_{o} \neq \varnothing$.

We depict an example in Figure 5.

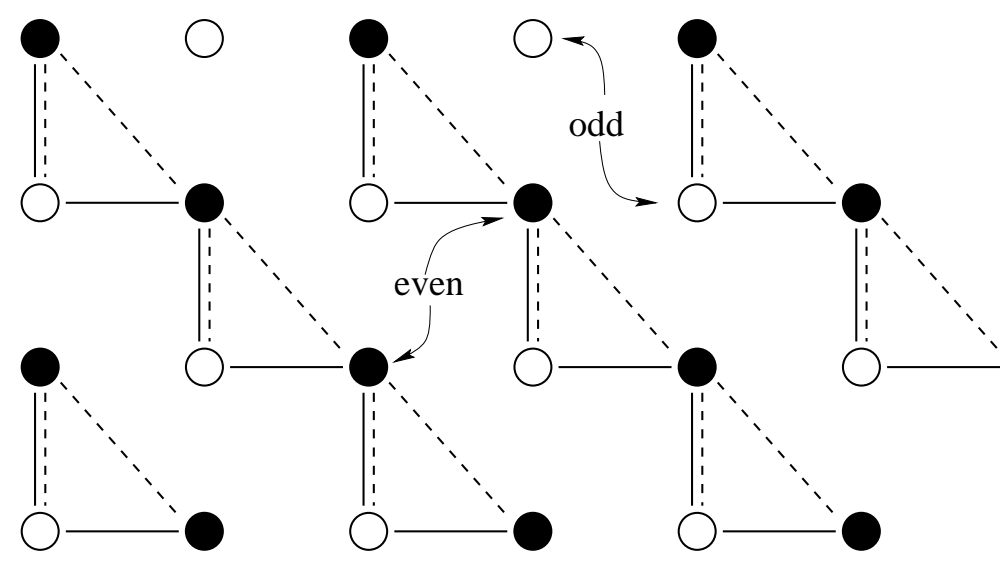

Fig. 5. Foreground adjacency (solid lines) and background adjacency (dashed lines) depend on whether a vector is odd or even. (Note that the figure has to be extended infinitely in the horizontal and vertical directions.)

Here

$$
A_{e}=\left[\begin{array}{lll}
\cdot & \cdot & \cdot \\
\bullet & \dot{ } & \cdot \\
\cdot & \bullet & \cdot
\end{array}\right.
$$$$
A_{o}=\begin{array}{ccc}
\cdot & \bullet & \cdot \\
\cdot & \dot{ } & \bullet \\
\cdot & \cdot & \cdot
\end{array}
$$$$
B_{e}=\begin{array}{lll}
\bullet & \cdot & \cdot \\
\cdot & \dot{-} & \cdot \\
\cdot & \bullet & \bullet
\end{array}
$$

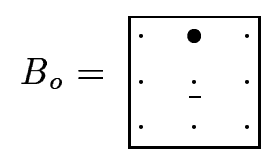


Every even point is a 0 -triple point and every odd point is a 1-triple point, and Corollary 5.8 says that $\psi^{3}=\psi^{2}$ in this case. It is easy to see that $\psi^{2} \neq \psi$. Namely, start with 0 's at the odd points and 1's at the even points. Then we get the sequence $01000 \cdots$ at the odd points and $10000 \cdots$ at the even points.

\section{Experiments and conclusion}

The annular filter given in (6.1) can also be applied to grey-scale images. In fact, every increasing operator on $\mathcal{P}(E)$ can be extended to grey-scale functions $F$ on $E$ by thresholding. The resulting operator is called a flat operator: see $[8]$ and $[1,2]$. For example, the extension of the dilation $X \mapsto X \oplus A$ is the operator $F \mapsto F \oplus A$ given by

$$
(F \oplus A)(x)=\sup _{a \in A} F(x-a) .
$$
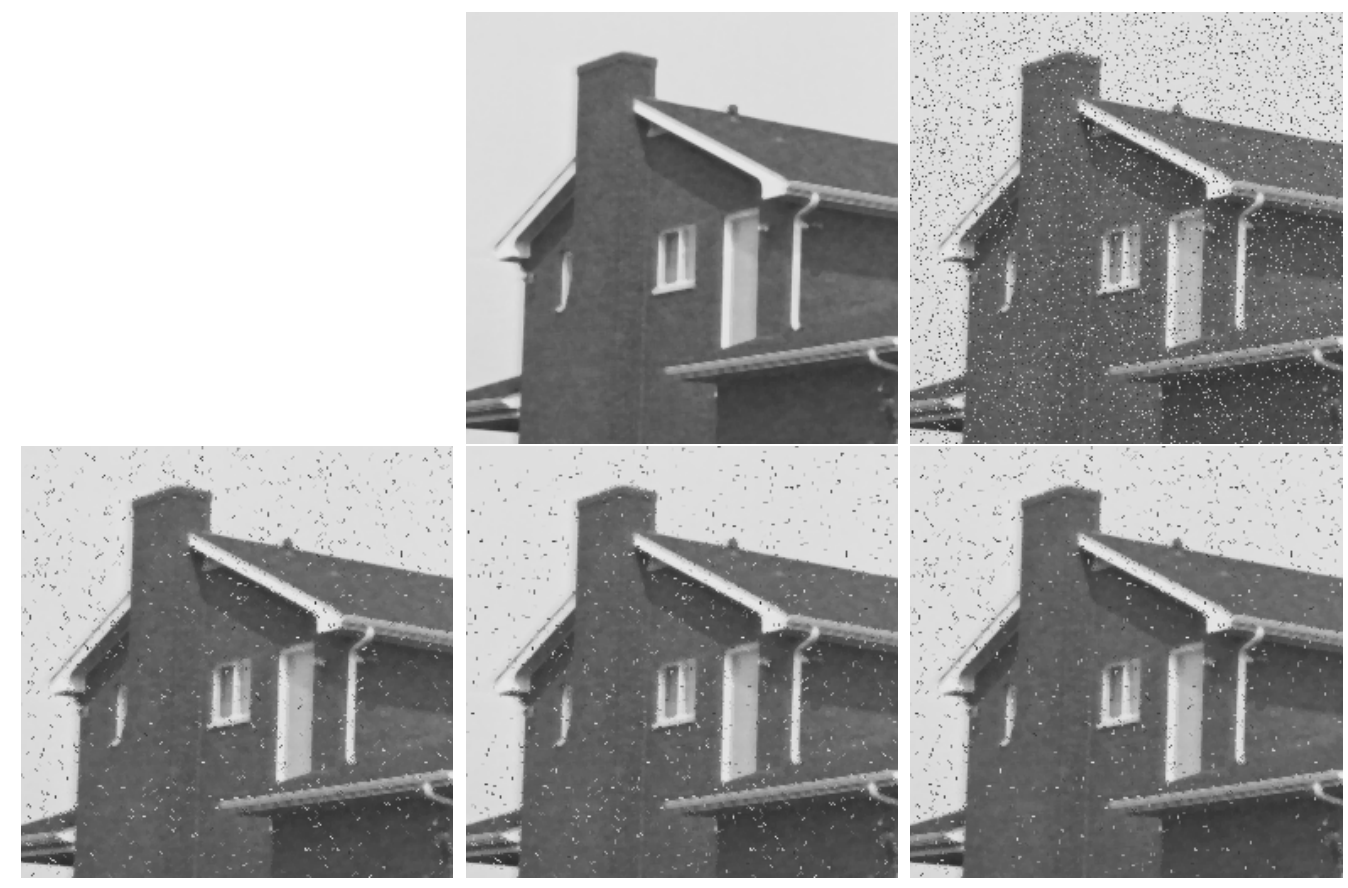

Fig. 6. Annular filters for grey-scale images. First row: image of a house and a noisy version which is used as input image. Bottom row, from left to right: self-dual annular filter with $A=B=S_{8}$; annular filter with $A=S_{8}$ and $B=S_{4}$; annular filter with $A=S_{4}$ and $B=S_{8}$.

In Figure 6 we illustrate three different annular filters. The input grey-scale image is a $256 \times 256$ house image in which approximately $10 \%$ of the pixels are distorted by salt-and-pepper noise. We denote by $S_{4}$ the set of four 4-neighbours of the origin and by $S_{8}$ the set of eight 8-neighbours. In Figure 7 we illustrate the same annular filters for a binary image, a thresholded version of the image in Figure 6. Observe that the second annular filter with $A=S_{8}$ and $B=S_{4}$ affects more background pixels (dark pixels in the grey-scale case depicted in Figure 6, and black pixels in Figure 7). Namely, if $B=S_{4}$, then a background (black) pixel is isolated if all of its 


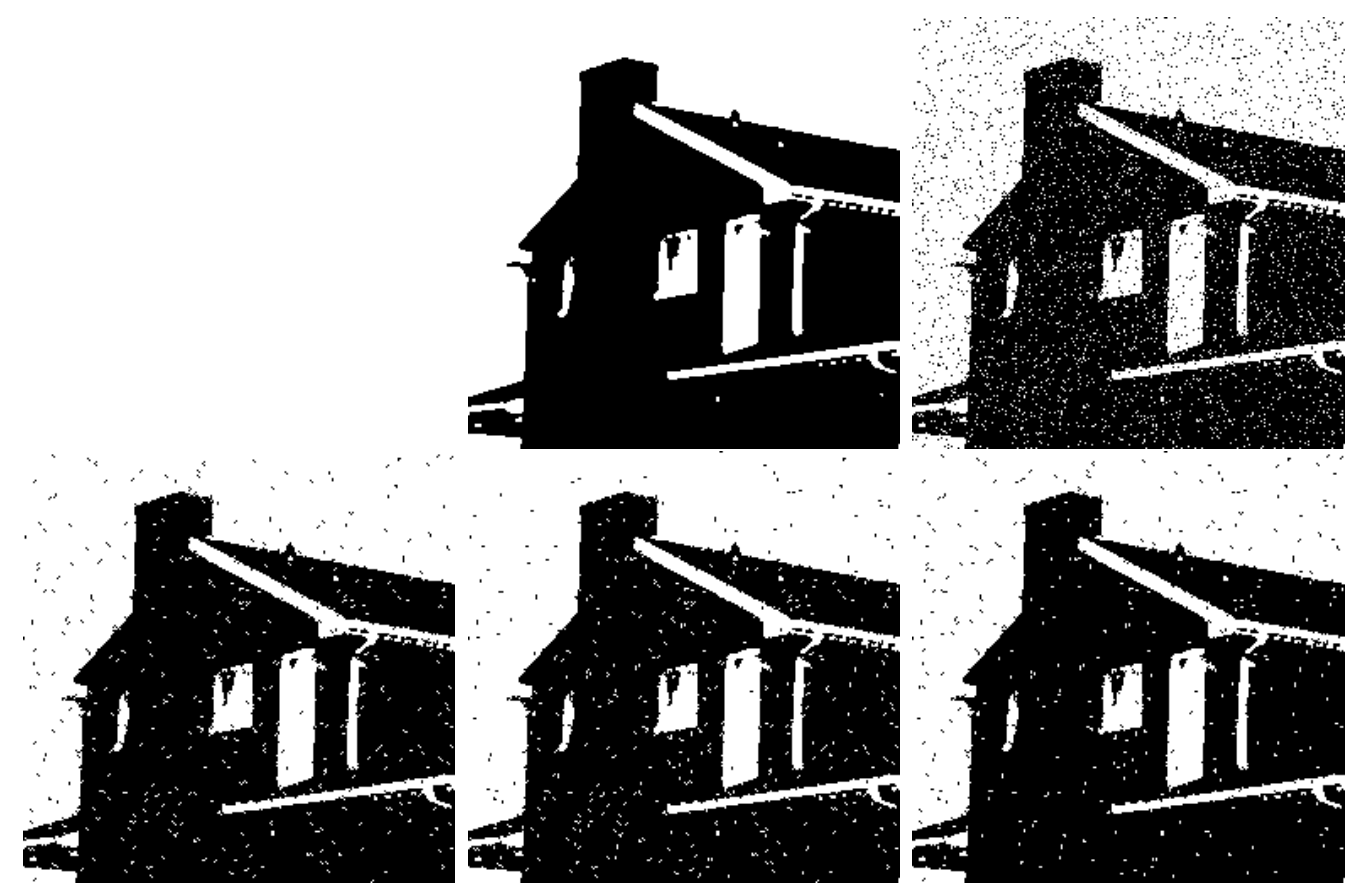

Fig. 7. Annular filters for binary images. These images are thresholds of those in Figure 6. Foreground pixels are white, background pixels are black.

for 4-neighbours lie in the foreground (white). If $B=S_{8}$, such a pixel is isolated if all its eight 8 -neighbours are white, which is a stronger condition.

In a forthcoming paper [7], we will generalize our results to lattices, and in particular complete lattices in which the so-called Basic Assumption [4] holds. In particular, we will derive there more general expressions for annular grey-scale filters where structuring elements are given by functions and not sets.

\section{Acknowledgment.}

The first author wants to thank Dan Schonfeld for some stimulating discussions.

\section{References.}

[1] Heijmans, H. J. A. M. Theoretical aspects of gray-level morphology. IEEE Transactions on Pattern Analysis and Machine Intelligence 13 (1991), 568-582.

[2] Heijmans, H. J. A. M. Morphological Image Operators. Academic Press, Boston, 1994.

[3] Heijmans, H. J. A. M. Self-dual morphological operators and filters. Journal of Mathematical Imaging and Vision 6, 1 (1996), 15-36.

[4] Heijmans, H. J. A. M., And Ronse, C. The algebraic basis of mathematical morphology part I: Dilations and erosions. Computer Vision, Graphics and Image Processing 50 (1990), $245-295$.

[5] Matheron, G. Random Sets and Integral Geometry. John Wiley and Sons, New York, 1975 .

[6] Ronse, C., And Heijmans, H. J. A. M. The algebraic basis of mathematical morphology - part II: Openings and closings. Computer Vision, Graphics and Image Processing: Image Understanding 54 (1991), 74-97. 
[7] Ronse, C., And Heijmans, H. J. A. M. A lattice-theoretical framework for annular filters in morphological image processing, In preparation.

[8] Serra, J. Image Analysis and Mathematical Morphology. Academic Press, London, 1982.

[9] Serra, J., Ed. Image Analysis and Mathematical Morphology. II: Theoretical Advances. Academic Press, London, 1988. 